\title{
ANALYSIS OF A MATHEMATICAL MODEL DESCRIBING NECROTIC TUMOR GROWTH
}

\author{
JOACHIM ESCHER, ANCA-VOICHITA MATIOC, AND BOGDAN-VASILE MATIOC
}

\begin{abstract}
In this paper we study a model describing the growth of necrotic tumors in different regimes of vascularisation. The tumor consists of a necrotic core of death cells and a surrounding nonnecrotic shell. The corresponding mathematical formulation is a moving boundary problem where both boundaries delimiting the nonnecrotic shell are allowed to evolve in time. We determine all radially symmetric stationary solutions of the problem and reduce the moving boundary problem into a nonlinear evolution. Parabolic theory provides us the perfect context in order to show local well-posed of the problem for small initial data.
\end{abstract}

\section{The MATHEMATiCAL MODEL}

In this paper we study a moving bondary problem describing the growth of a necrotic tumor in the absence of inhibitor. The model purposed initially in $[4,15,17]$, was reformulated by using algebraic manipulations $[5,12]$ to describe evolution of tumors in all regimes of vascularisation. Nevertheless, the analysis in $[5,12]$ is simplified by the assumption that the tumor core is nonnecrotic. This aspect is considered into our modeling, where following $[10,17,18]$, we assume that the tumor consists of a core of death cells (necrotic core) and a shell of life-proliferating cells surrounding the core (nonnecrotic shell). The blood supply provides the nonnecrotic region with nutrients, while there is no blood supply in the necrotic region and the concentration of nutrients is at a constant level which cannot sustain cell proliferation. However the model presented here includes two moving boundaries, one parametrising the boundary of the necrotic core and one for the outer boundary of the tumor, both of them having infinitely many degrees of freedom. This fact makes the problem more involved in comparison to other models which either neglect the necrotic core $[3,6-10]$ or consider only the radially symmetric problem when the tumors are annular domains $[10,17,18]$.

2000 Mathematics Subject Classification. 35K55; 35R35; 35R37.

Key words and phrases. Radially symmetric; Stationary solution; Classical solution; Fourier multiplier. 
The mathematical model is given by the following system of equations

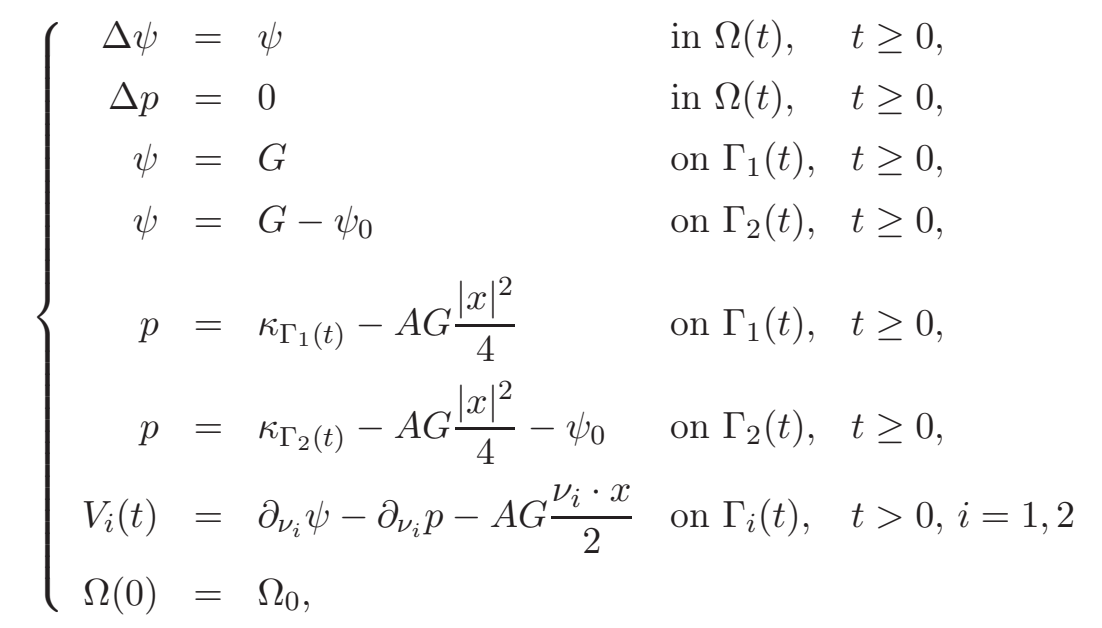

where $\Omega(t) \subset \mathbb{R}^{2}$ is the domain occupied by the nonnecrotic shell, $\psi$ is the rate at which nutrient is added to $\Omega(t)$, over the outer boundary $\Gamma_{1}(t)$, by the vascularisation, $p$ is the pressure, $\Gamma_{2}(t)$ is the interior boundary enclosing the necrotic core, $\nu_{i}$ the outward orientated normal and $\kappa_{\Gamma_{i}(t)}$ the curvature of $\Gamma_{i}(t), i=1,2$. By convention, $\kappa_{\Gamma_{1}(t)}$ is positive and $\kappa_{\Gamma_{2}(t)}$ negative if $\Gamma_{i}(t)$, $1 \leq i \leq 2$ are close to a circle. Moreover, $V_{i}(t)$ stands for the normal velocity of $\Gamma_{i}(t)$, the constants $A, G \in \mathbb{R}$ have biological relevancy being related to cell proliferation, cell apoptosis, and vascularisation. The scalar $\psi_{0}>0$ is linked with the constant nutrient concentration assumed within the necrotic region. The initial tumor domain is given by $\Omega_{0}$.

For a precise deduction of the system (1.1) and its biological meaning we refer to $[5,12]$, the only difference to the model presented there being the consideration of the interior necrotic region bounded by $\Gamma_{2}(t)$.

The first main result of this paper is the following theorem:

Theorem 1.1. Given $\left(R_{1}, R_{2}\right) \in(0, \infty)^{2}$ with $R_{2}<R_{1}$, let $\psi_{0}^{c}$ be the constant defined by (2.8). There exists $A \in \mathbb{R}$ and $G \in \mathbb{R} \backslash\{0\}$, such that the annulus

$$
A\left(R_{1}, R_{2}\right):=\left\{x \in \mathbb{R}^{2}: R_{2}<|x|<R_{1}\right\},
$$

is a stationary solution of problem (1.1) provided $\psi_{0} \neq \psi_{0}^{c}$. Moreover, $A$ and $G$ are uniquelly determined by $R_{1}, R_{2}$, and $\psi_{0}$.

If $G=0$, then problem (1.1) has no radially symmetric stationary solutions.

In contrast to $[5,12]$, where the radially symmetric stationary tumors are circles with radius which depends only on the constant $A$, the radii of the stationary annular tumors found in Theorem 1.1 depend on both constants $A$ and $G$, but also on $\psi_{0}$, cf. (2.9).

In order to prove local-well-posedness of the moving boundary problem (1.1) the well-posedness of system (1.1) (see Theorem 1.2 below) we introduce first a parametrisation for the interfaces $\Gamma_{1}(t)$ and $\Gamma_{2}(t)$, which are the main 


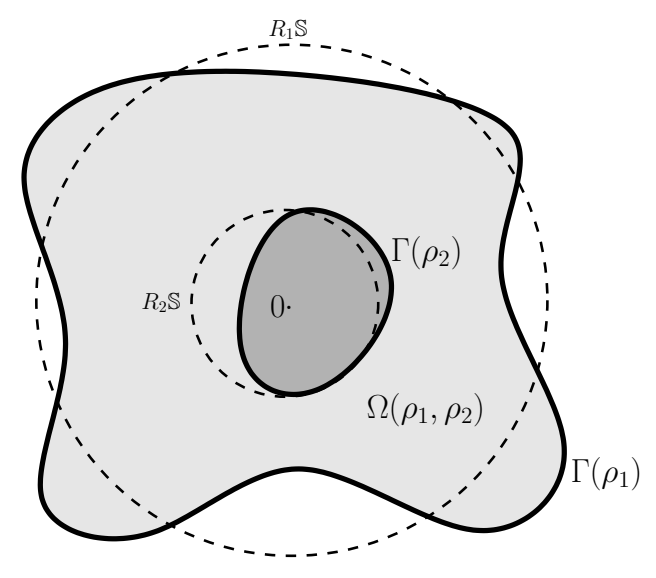

FiguRE 1. Parametrisation of the tumor domain

unknowns of system (1.1). Let $0<R_{2}<R_{1}$ be given and fix $\alpha \in(0,1)$. We set

$$
\mathcal{V}:=\left\{\rho \in h^{4+\alpha}(\mathbb{S}):\|\rho\|_{C(\mathbb{S})}<a\right\}
$$

where

$$
a<\frac{R_{1}-R_{2}}{R_{1}+R_{2}} .
$$

The small Hölder space $h^{m+\beta}(\mathbb{S}), \beta \in(0,1)$ and $m \in \mathbb{N}$, is defined as the completion of the smooth functions in $C^{m+\beta}(\mathbb{S})$. Each pair $\left(\rho_{1}, \rho_{2}\right) \in \mathcal{V}^{2}$ parametrises a $C^{4+\alpha}$-domain

$$
\Omega\left(\rho_{1}, \rho_{2}\right):=\left\{y \in \mathbb{R}^{2}: R_{2}\left(1+\rho_{2}(y /|y|)\right)<|y|<R_{1}\left(1+\rho_{1}(y /|y|)\right)\right\} .
$$

The condition on $a$ ensures that the boundary portions of $\Omega\left(\rho_{1}, \rho_{2}\right)$

$$
\Gamma\left(\rho_{i}\right):=\left\{x:|x|=R_{i}\left(1+\rho_{i}(x /|x|)\right)\right\},
$$

$i=1,2$, are disjoint (see Figure 1$)$ for any choice of $\left(\rho_{1}, \rho_{2}\right) \in \mathcal{V}^{2}$. They can be seen to be zero level sets, $\Gamma\left(\rho_{i}\right)=N_{\rho_{i}}{ }^{-1}(0)$, where $N_{\rho_{i}}: \mathbb{R}^{2} \backslash\{0\} \rightarrow \mathbb{R}, i=$ 1,2 , are defined by

$$
N_{\rho_{i}}(x)=|x|-R_{i}-R_{i} \rho_{i}(x /|x|), \quad x \neq 0 .
$$

Hence, the outward unit normal at $\partial \Omega\left(\rho_{1}, \rho_{2}\right)$ is given by

$$
\nu_{\rho_{1}}=\frac{\nabla N_{\rho_{1}}}{\left|\nabla N_{\rho_{1}}\right|} \text { on } \Gamma\left(\rho_{1}\right) \text {, and } \nu_{\rho_{2}}=-\frac{\nabla N_{\rho_{2}}}{\left|\nabla N_{\rho_{2}}\right|} \text { on } \Gamma\left(\rho_{2}\right) \text {. }
$$

If $\left(\rho_{1}, \rho_{2}\right):[0, T] \rightarrow \mathcal{V}^{2}$ describe the motion of the tumor, then we can express the normal velocity of both boundary components in terms of $\rho_{i}$ by the formula

$$
V_{1}(t)=-\frac{\partial_{t} N_{\rho_{1}}}{\left|\nabla N_{\rho_{1}}\right|} \text { on } \Gamma\left(\rho_{1}(t)\right) \text {, and } V_{2}(t)=\frac{\partial_{t} N_{\rho_{2}}}{\left|\nabla N_{\rho_{2}}\right|} \text { on } \Gamma\left(\rho_{2}(t)\right) .
$$


With this notation, system (1.1) becomes a problem with $\rho_{1}$ and $\rho_{2}$ as unknowns:

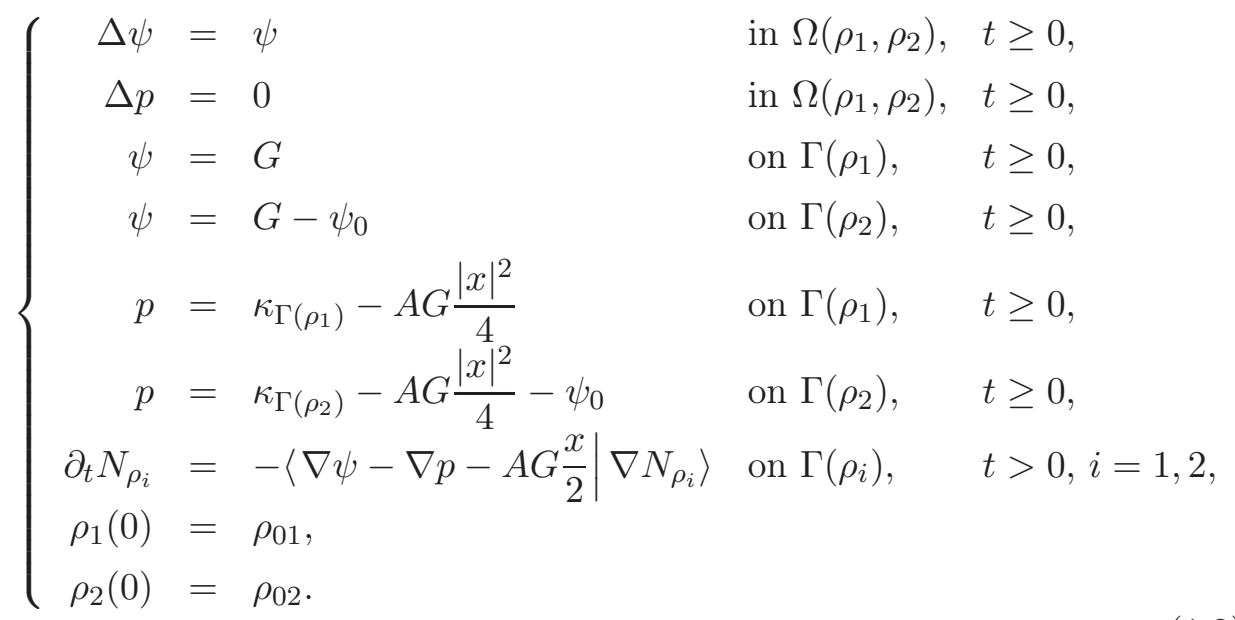

A pair $\left(\rho_{1}, \rho_{2}, \psi, p\right)$ is called classical solution of $(1.1)$ on $[0, T], T>0$, if

$$
\begin{gathered}
\rho_{i} \in C([0, T], \mathcal{V}) \cap C^{1}\left([0, T], h^{1+\alpha}(\mathbb{S})\right), i=1,2, \\
\psi(t, \cdot), p(t, \cdot) \in b u c^{2+\alpha}\left(\Omega\left(\rho_{1}(t), \rho_{2}(t)\right)\right), t \in[0, T],
\end{gathered}
$$

and if $\left(\rho_{1}, \rho_{2}, \psi, p\right)$ solves $(1.2)$ pointwise. Given $U \subset \mathbb{R}^{2}$ open, we set $b u c^{2+\alpha}(U)$ to be the closure of the the smooth functions with bounded and uniformly continuous derivatives $B U C^{\infty}(U)$ within $B U C^{2+\alpha}(U)$ (if $U$ is also bounded then $B U C^{2+\alpha}(U)=C^{2+\alpha}(\bar{U})$.)

Concerning well-posedness of system (1.1), our second main result states that problem (1.1) possesses a unique solution provided that initially the tumor is close to an annulus (which is not necessarily a stationary solution).

Theorem 1.2 (Local well-posedness). Let $0<R_{2}<R_{1}$, and $\left(A, G, \psi_{0}\right) \in \mathbb{R}^{3}$ be given.

There exists an open neighbourhood $\mathcal{O} \subset \mathcal{V}$ such that for all $\left(\rho_{1}, \rho_{2}\right) \in \mathcal{O}^{2}$, problem (1.2) possesses a unique classical solution defined on a maximal time interval $\left[0, T\left(\rho_{01}, \rho_{02}\right)\right)$ and which satisfies $\left(\rho_{1}, \rho_{2}\right)(t) \in \mathcal{O}^{2}$ for all $t \in$ $\left[0, T\left(\rho_{01}, \rho_{02}\right)\right)$.

The outline of this paper is as follows: we study in Section 2 the radially symmetric free boundary problem which describes the stationary solutions of problem (1.1) and prove Theorem 1.1. In the last section of this paper we prove the local well-posedness result Theorem 1.2.

\section{RADIALLY SYMMETRIC STATIONARY SOLUTIONS}

We determine in this section the radially symmetric steady-state solutions of (1.1), situation when the nonnecrotic shell is a steady annulus.

The most simple situation is the case $G=0$, when the problem is invariant under translation and rotations.. Then, the annulus $A\left(R_{1}, R_{2}\right)$ centred in 
zero with radii $R_{1}>R_{2}$, is a stationary solution of system (1.1) if and only if

$$
p^{\prime}\left(R_{i}\right)=\psi^{\prime}\left(R_{i}\right), \quad i=1,2,
$$

where $p$ is the solution of the problem

$$
\left\{\begin{array}{rlr}
p^{\prime \prime}+\frac{1}{r} p^{\prime} & =0, & R_{2}<r<R_{1}, \\
p\left(R_{1}\right) & =R_{1}{ }^{-1}-A G R_{1}^{2} / 4, & \\
p\left(R_{2}\right) & =-R_{2}{ }^{-1}-A G R_{2}^{2} / 4-\psi_{0}, &
\end{array}\right.
$$

when $G=0$. System (2.1) corresponds to the Dirichlet problem for the pressure $p$ in (1.1) (the second, fifth, and sixth equations of (1.1)), where we used polar coordinates when expressing the Laplacian. Notice that the boundary data are constants, thus $p$ depends only on $r$, the distance to the origin.

Given $G \in \mathbb{R}$, the solution of (2.1) is given by the relation $p(r)=a_{R_{1} R_{2}} \ln (r)+$ $b_{R_{1} R_{2}}, r_{2} \leq|r| \leq R_{1}$, with

$$
\begin{aligned}
& a_{R_{1} R_{2}}=\frac{R_{1}^{-1}+R_{2}^{-1}+A G\left(R_{2}^{2}-R_{1}^{2}\right) / 4+\psi_{0}}{\ln \left(R_{1} / R_{2}\right)}, \\
& b_{R_{1} R_{2}}=R_{1}^{-1}-A G R_{1}^{2} / 4-a_{R_{1} R_{2}} \ln \left(R_{1}\right) .
\end{aligned}
$$

Furthermore, $\psi$ is the solution of the problem

$$
\left\{\begin{aligned}
\psi^{\prime \prime}+\frac{1}{r} \psi^{\prime}-\psi & =0, \quad R_{2}<r<R_{1}, \\
\psi\left(R_{1}\right) & =G, \\
\psi\left(R_{2}\right) & =G-\psi_{0},
\end{aligned}\right.
$$

when $G=0$. Also, for fixed $G \in \mathbb{R}$, the solution of $(2.2)$ can be written as linear combination of modified Bessel functions of first and second kind $\psi=c_{R_{1} R_{2}}^{1} I_{0}+c_{R_{1} R_{2}}^{2} K_{0}$, with scalars

$$
\begin{aligned}
c_{R_{1} R_{2}}^{1} & =\frac{G K_{0}\left(R_{2}\right)+\left(\psi_{0}-G\right) K_{0}\left(R_{1}\right)}{I_{0}\left(R_{1}\right) K_{0}\left(R_{2}\right)-I_{0}\left(R_{2}\right) K_{0}\left(R_{1}\right)}, \\
c_{R_{1} R_{2}}^{2} & =\frac{-G I_{0}\left(R_{2}\right)-\left(\psi_{0}-G\right) I_{0}\left(R_{1}\right)}{I_{0}\left(R_{1}\right) K_{0}\left(R_{2}\right)-I_{0}\left(R_{2}\right) K_{0}\left(R_{1}\right)} .
\end{aligned}
$$

Consequently, $A\left(R_{1}, R_{2}\right)$ is a steady-state solution of (1.1) when $G=0$ if and only if

$$
\frac{\frac{1}{R_{1}}+\frac{1}{R_{2}}+\psi_{0}}{\ln \left(R_{1} / R_{2}\right)} \frac{1}{R_{i}}=\psi_{0} \frac{K_{0}\left(R_{1}\right) I_{1}\left(R_{i}\right)+I_{0}\left(R_{1}\right) K_{1}\left(R_{i}\right)}{I_{0}\left(R_{1}\right) K_{0}\left(R_{2}\right)-I_{0}\left(R_{2}\right) K_{0}\left(R_{1}\right)}, \quad i=1,2,
$$

where we used the relations $I_{0}^{\prime}=I_{1}$ and $K_{0}^{\prime}=-K_{1}$. It follows then easily that the system consisting of the equations (2.3) has solutions $\left(R_{1}, R_{2}\right)$ with 
$R_{1}>R_{2}$ exactly when

$$
\frac{R_{2}}{R_{1}}=\frac{K_{0}\left(R_{1}\right) I_{1}\left(R_{1}\right)+I_{0}\left(R_{1}\right) K_{1}\left(R_{1}\right)}{K_{0}\left(R_{1}\right) I_{1}\left(R_{2}\right)+I_{0}\left(R_{1}\right) K_{1}\left(R_{2}\right)} .
$$

Equation (2.4) is obtained by expressing $\psi_{0}$ in both relations (2.3) and setting them to be equal. We show now that equality holds in the relation above only when $R_{1}=R_{2}$. Indeed, fix $R_{1}>0$ and consider the auxiliary function $g:\left(0, R_{1}\right] \rightarrow \mathbb{R}$ with

$g(x)=K_{0}\left(R_{1}\right) x I_{1}(x)+I_{0}\left(R_{1}\right) x K_{1}(x)-R_{1}\left(K_{0}\left(R_{1}\right) I_{1}\left(R_{1}\right)+I_{0}\left(R_{1}\right) K_{1}\left(R_{1}\right)\right)$

for $0<x \leq R_{1}$. Obviously $g\left(R_{1}\right)=0$. If we show that the derivative $g^{\prime}$ has constant sign on $\left(0, R_{1}\right]$ then we are done, that is there is no positive $R_{2}<R_{1}$ such that $\left(R_{1}, R_{2}\right)$ solves (2.4). Well-known properties of the modified Bessel functions (see [2]) lead to

$$
\begin{aligned}
g^{\prime}(x)= & K_{0}\left(R_{1}\right) I_{1}(x)+I_{0}\left(R_{1}\right) K_{1}(x) \\
& +K_{0}\left(R_{1}\right) x\left(I_{0}(x)-(1 / x) I_{1}(x)\right)+I_{0}\left(R_{1}\right) x\left(-K_{0}(x)-(1 / x) K_{1}(x)\right) \\
= & x\left(I_{0}(x) K_{0}\left(R_{1}\right)-I_{0}\left(R_{1}\right) K_{0}(x)\right)<0
\end{aligned}
$$

for all $x \in\left(0, R_{1}\right)$. That the last expression is negative is a consequence of the following facts: $I_{0}$ and $K_{0}$ are both positive functions, $I_{0}$ is strictly increasing, and $K_{0}$ is strictly decreasing. Hence, problem (1.1) has no radially symmetric stationary when $G=0$.

Let now $G \neq 0$. In this case $A\left(R_{1}, R_{2}\right)$ is a steady-state solution of (1.1) exactly when

$$
\psi_{R_{1} R_{2}}^{\prime}\left(R_{i}\right)-p^{\prime}\left(R_{i}\right)-A G \frac{R_{i}}{2}=0, \quad i=1,2 .
$$

Using again the relations $I_{0}^{\prime}=I_{1}$ and $K_{0}^{\prime}=-K_{1}$, the identities (2.5) re-write

$$
c_{R_{1} R_{2}}^{1} I_{1}\left(R_{i}\right)-c_{R_{1} R_{2}}^{2} K_{1}\left(R_{i}\right)-a_{R_{1} R_{2}} \frac{1}{R_{i}}-A G \frac{R_{i}}{2}=0, \quad i=1,2,
$$

which seem to be very involved as expressions of variables $R_{1}$ and $R_{2}$ when trying to solve the system consisting of both of them. However, they can be viewed as equations for $A$ and $G$

$$
a_{i} G+b_{i} A G=c_{i}, \quad i=1,2,
$$

with coefficients $a_{i}, b_{i}$, and $c_{i}$ given by:

$$
\begin{aligned}
a_{i} & :=\frac{\left(K_{0}\left(R_{2}\right)-K_{0}\left(R_{1}\right)\right) I_{1}\left(R_{i}\right)-\left(I_{0}\left(R_{1}\right)-I_{0}\left(R_{2}\right)\right) K_{1}\left(R_{i}\right)}{I_{0}\left(R_{1}\right) K_{0}\left(R_{2}\right)-I_{0}\left(R_{2}\right) K_{0}\left(R_{1}\right)}, \\
b_{i} & :=\frac{R_{1}^{2}-R_{2}^{2}}{4 \ln \left(R_{1} / R_{2}\right)} \frac{1}{R_{i}}-\frac{R_{i}}{2}, \\
c_{i} & :=-\psi_{0} \frac{K_{0}\left(R_{1}\right) I_{1}\left(R_{i}\right)+I_{0}\left(R_{1}\right) K_{1}\left(R_{i}\right)}{I_{0}\left(R_{1}\right) K_{0}\left(R_{2}\right)-I_{0}\left(R_{2}\right) K_{0}\left(R_{1}\right)}+\frac{R_{1}^{-1}+R_{2}^{-1}+\psi_{0}}{\ln \left(R_{1} / R_{2}\right)} \frac{1}{R_{i}} .
\end{aligned}
$$



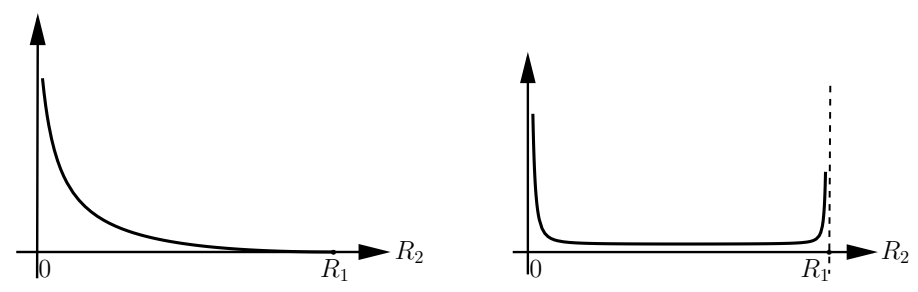

Figure 2. The expression $a_{1} b_{2}-a_{2} b_{1}$ and $\psi_{0}^{c}$, for fixed $R_{1}$, as a function of the variable $R_{2} \in\left(0, R_{1}\right)$.

The system of equations (2.6) has a (unique) solution $(A, G)$ with $G \neq 0$ provided that

$$
\begin{aligned}
& a_{1} b_{2}-a_{2} b_{1} \neq 0, \quad c_{1} b_{2}-c_{2} b_{1} \neq 0, \\
& \text { and } \quad c_{1} \neq 0 \quad \text { or } \quad c_{2} \neq 0 .
\end{aligned}
$$

The computation done for the case $G=0$ shows that $c_{1}$ and $c_{2}$ cannot be simultaneously zero when $R_{2}<R_{1}$. For fixed $R_{1}>0$ we may see the expression $a_{1} b_{2}-a_{2} b_{1}$ as a function of $R_{2} \in\left(0, R_{1}\right)$. This function is decreasing with respect to $R_{2}$ (see Figure 2), thus $a_{1} b_{2}=a_{2} b_{1}$ only when $R_{1}=R_{2}$. Furthermore, $b_{1} c_{2}=b_{2} c_{1}$ if and only if $\psi_{0}=\psi_{0}^{c}$, where

$$
\psi_{0}^{c}:=\frac{\left(b_{1} / R_{1}-b_{2} / R_{2}\right) \frac{1 / R_{1}+1 / R_{2}}{\ln \left(R_{1} / R_{2}\right)}}{\frac{K_{0}\left(R_{1}\right)\left(b_{1} I_{1}\left(R_{2}\right)-b_{2} I_{1}\left(R_{1}\right)\right)+I_{0}\left(R_{1}\right)\left(b_{1} K_{1}\left(R_{2}\right)-b_{2} K_{1}\left(R_{1}\right)\right)}{I_{0}\left(R_{1}\right) K_{0}\left(R_{2}\right)-I_{0}\left(R_{2}\right) K_{0}\left(R_{1}\right)}+\frac{R_{1}^{2}-R_{2}^{2}}{2 R_{1} R_{2} \ln \left(R_{1} / R_{2}\right)}} .
$$

It is not difficult to see that the numerator of the fraction is negative, and the same holds true for the denominator, implying that $\psi_{0}^{c}>0$. We plotted in Figure 2 the expression on the right hand side of (2.8) for fixed $R_{1}>0$ in dependence of $R_{2} \in\left(0, R_{1}\right)$. Consequently, $A\left(R_{1}, R_{2}\right)$ is a stationary solution of (1.1) if and only if $\psi_{0}$ is not the critical constant given by (2.8) and

$$
A=\frac{a_{1} c_{2}-a_{2} c_{1}}{c_{1} b_{2}-c_{2} b_{1}}, \quad G=\frac{c_{1} b_{2}-c_{2} b_{1}}{a_{1} b_{2}-a_{2} b_{1}} .
$$

This proves Theorem 1.1.

\section{The MOVING BOUNDARY PROBLEM}

This last section is dedicated entirely to the proof of our secon main result, Theorem 1.2. In order to prove well-posedness of problem (1.1) in the context defined in the introduction we transform first (1.2) into a problem on the fixed domain $\Omega:=\Omega(0,0)$, with boundary $\Gamma_{1}:=R_{1} \mathbb{S}$ and $\Gamma_{2}:=R_{2} \mathbb{S}$. This transformation will allow us to introduce solution operators related to problem (1.2) and which will enable us to reduce system (1.2) into an abstract nonlinear evolution equation for the pair $\left(\rho_{1}, \rho_{2}\right)$. 
Pick therefore $0<R_{2}<R_{1},\left(A, G, \psi_{0}\right) \in \mathbb{R}^{3}$, and $\alpha \in(0,1)$. Given $\left(\rho_{1}, \rho_{2}\right) \in \mathcal{V}^{2}$, we define the mapping $\Theta_{\rho_{1}, \rho_{2}}: \Omega \rightarrow \Omega\left(\rho_{1}, \rho_{2}\right)$ by the relation

$$
\Theta_{\rho_{1}, \rho_{2}}(x)=\frac{\left(R_{1}-|x|\right) R_{2}\left(1+\rho_{2}(x /|x|)\right)+\left(|x|-R_{2}\right) R_{1}\left(1+\rho_{1}(x /|x|)\right)}{R_{1}-R_{2}} \frac{x}{|x|}
$$

for $x \in \Omega$. One can easily check that $\Theta_{\rho_{1}, \rho_{2}}$ is an diffeomorphism, i.e. $\Theta_{\rho_{1}, \rho_{2}} \in \operatorname{Diff}^{4+\alpha}\left(\Omega, \Omega\left(\rho_{1}, \rho_{2}\right)\right)$, which maps $\Gamma_{i}$ onto $\Gamma\left(\rho_{i}\right), i=1,2$. Using this diffeomorphism, we define the transformed operators

$\mathcal{A}\left(\rho_{1}, \rho_{2}\right): b u c^{2+\alpha}(\Omega) \rightarrow b u c^{\alpha}(\Omega), \quad \mathcal{A}\left(\rho_{1}, \rho_{2}\right) v:=\Delta\left(v \circ \Theta_{\rho_{1}, \rho_{2}}^{-1}\right) \circ \Theta_{\rho_{1}, \rho_{2}}-v$, which is an elliptic operator depending analytically on $\left(\rho_{1}, \rho_{2}\right)$, i.e.

$$
\mathcal{A} \in C^{\omega}\left(\mathcal{V}^{2}, \mathcal{L}\left(b u c^{2+\alpha}(\Omega), b u c^{\alpha}(\Omega)\right)\right),
$$

respectively the trace operators $\mathcal{B}_{i}: \mathcal{V}^{2} \times\left(b u c^{2+\alpha}(\Omega)\right)^{2} \rightarrow h^{1+\alpha}(\mathbb{S})$ by

$$
\mathcal{B}_{i}\left(\rho_{1}, \rho_{2}, v, q\right):=\frac{1}{R_{i}} \mathcal{C}_{i}\left(\rho_{1}, \rho_{2}\right) v-\frac{1}{R_{i}} \mathcal{C}_{i}\left(\rho_{1}, \rho_{2}\right) q-\mathcal{D}_{i}\left(\rho_{1}, \rho_{2}\right) .
$$

Given $\left(\rho_{1}, \rho_{2}\right) \in \mathcal{V}^{2}$, the linear operators $\left.\mathcal{C}_{i}\left(\rho_{1}, \rho_{2}\right) \in \mathcal{L}\left(b u c^{2+\alpha}(\Omega)\right), h^{1+\alpha}(\mathbb{S})\right)$, $i=1,2$, are given by

$$
\mathcal{C}_{i}\left(\rho_{1}, \rho_{2}\right) v(y):=\left\langle\nabla\left(v \circ \Theta_{\rho_{1}, \rho_{2}}^{-1}\right) \mid \nabla N_{\rho_{i}}\right\rangle \circ \Theta_{\rho_{1}, \rho_{2}}\left(R_{i} y\right)
$$

for $v \in b u c^{2+\alpha}(\Omega)$ and $y \in \mathbb{S}$. Moreover,

$$
\mathcal{D}_{i}\left(\rho_{1}, \rho_{2}\right):=-\frac{A G}{R_{i}}\left\langle\frac{x}{2} \mid \nabla N_{\rho_{i}}\right\rangle \circ \Theta_{\rho_{1}, \rho_{2}}\left(R_{i} y\right) .
$$

The operators $\mathcal{C}_{i}$ and $\mathcal{D}_{i}, i=1,2$, depend analytically on $\left(\rho_{1}, \rho_{2}\right)$ too,

$$
\left.\mathcal{C}_{i} \in C^{\omega}\left(\mathcal{V}^{2}, \mathcal{L}\left(b u c^{2+\alpha}(\Omega)\right), h^{1+\alpha}(\mathbb{S})\right)\right) \text { and } \mathcal{D}_{i} \in C^{\omega}\left(\mathcal{V}^{2}, h^{1+\alpha}(\mathbb{S})\right) .
$$

Having defined this operators we may re-write now (1.2) in an equivalent form. Namely, if $\left(\rho_{1}, \rho_{2}, \psi, p\right)$ is a solution of (1.2), $v:=\psi \circ \Theta_{\rho_{1}, \rho_{2}}$, and $q:=p \circ \Theta_{\rho_{1}, \rho_{2}}$, then the tupel $\left(\rho_{1}, \rho_{2}, v, q\right)$ solves the following system

$$
\left\{\begin{aligned}
\mathcal{A}\left(\rho_{1}, \rho_{2}\right) v & =0 & & \text { in } \Omega, \quad t \geq 0 \\
\mathcal{A}\left(\rho_{1}, \rho_{2}\right) q & =0 & & \text { in } \Omega, \quad t \geq 0, \\
v & =G & & \text { on } \Gamma_{1}, \quad t \geq 0 \\
v & =G-\psi_{0} & & \text { on } \Gamma_{2}, \quad t \geq 0 \\
q & =\frac{1}{R_{1}} \kappa\left(\rho_{1}\right)-\frac{A G R_{1}^{2}}{4}\left(1+\rho_{1}\right)^{2} & & \text { on } \Gamma_{1}, \quad t \geq 0 \\
q & =-\frac{1}{R_{2}} \kappa\left(\rho_{2}\right)-\frac{A G R_{2}^{2}}{4}\left(1+\rho_{2}\right)^{2}-\psi_{0} & & \text { on } \Gamma_{2}, \quad t \geq 0 \\
\partial_{t} \rho_{i} & =\mathcal{B}_{i}\left(\rho_{1}, \rho_{2}, v, q\right) & & \\
\rho_{1}(0) & =\rho_{01}, & &
\end{aligned}\right.
$$


where $\kappa: \mathcal{V} \rightarrow h^{2+\alpha}(\mathbb{S})$ is defined by

$$
\kappa(\rho):=\frac{(1+\rho)^{2}+2 \rho^{\prime 2}-(1+\rho) \rho^{\prime \prime}}{\left((1+\rho)^{2}+\rho^{2}\right)^{3 / 2}}, \quad \rho \in \mathcal{V},
$$

and we identified functions on $\Gamma_{i}$ with those on $\mathbb{S}, i=1,2$, via the diffeomorphisms $\left[\mathbb{S} \ni y \mapsto R_{i} y \in \Gamma_{i}\right]$.

Though the problem becomes more involved (the diffeomorphism introduces additionally nonlinearities), (3.3) has the advantage that the sets where the differential equations and the boundary conditions are imposed do not change with time. It is convenient now to introduce solution operators to Dirichlet problem closely related to system (3.3).

Lemma 3.1. Given $\left(\rho_{1}, \rho_{2}\right) \in \mathcal{V}^{2}$, we let $\mathcal{T}\left(\rho_{1}, \rho_{2}\right), \mathcal{S}\left(\rho_{1}, \rho_{2}\right) \in$ buc ${ }^{2+\alpha}(\Omega)$ denote the unique solution of

$$
\left\{\begin{aligned}
\mathcal{A}\left(\rho_{1}, \rho_{2}\right) v & =v & & \text { in } \Omega, \\
v & =G & & \text { on } \Gamma_{1}, \\
v & =G-\psi_{0} & & \text { on } \Gamma_{2},
\end{aligned}\right.
$$

and

$$
\left\{\begin{aligned}
\mathcal{A}\left(\rho_{1}, \rho_{2}\right) q & =0 & & \text { in } \Omega, \\
q & =\frac{1}{R_{1}} \kappa\left(\rho_{1}\right)-\frac{A G R_{1}^{2}}{4}\left(1+\rho_{1}\right)^{2} & & \text { on } \Gamma_{1}, \\
q & =-\frac{1}{R_{2}} \kappa\left(\rho_{2}\right)-\frac{A G R_{2}^{2}}{4}\left(1+\rho_{2}\right)^{2}-\psi_{0} & & \text { on } \Gamma_{2},
\end{aligned}\right.
$$

respectively. The operators $\mathcal{T}$ and $\mathcal{S}$ depend analytically on $\left(\rho_{1}, \rho_{2}\right)$.

Proof. Given $\left(\rho_{1}, \rho_{2}\right) \in \mathcal{V}^{2}$, the Dirichlet problems (3.4) and (3.5) are uniquelly solvable, cf. [16, Theorem 6.14]. Moreover, since $\mathcal{A}$ and $\kappa$ depend analytically on their variables we deduce that also $\mathcal{T}$ and $\mathcal{S}$ do that. We may take now into consideration that $\mathcal{T}$ and $\mathcal{S}$ map both the smooth functions into $B U C^{2+\alpha}(\Omega)$ and conclude that their range is contained in $b u c^{2+\alpha}(\Omega)$.

With this definion (3.3) reduces to the following evolution equation

$$
\partial_{t} X=\Phi(X) \quad X(0)=X_{0},
$$

where $X:=\left(\rho_{1}, \rho_{2}\right), X_{0}:=\left(\rho_{01}, \rho_{02}\right)$, and $\Phi:=\left(\Phi_{1}, \Phi_{2}\right)$. The components of the nonlocal and nonlinear operator $\Phi$ are defined as follows

$$
\Phi_{i}\left(\rho_{1}, \rho_{2}\right):=\mathcal{B}_{i}\left(\rho_{i}, \mathcal{T}\left(\rho_{1}, \rho_{2}\right), \mathcal{S}\left(\rho_{1}, \rho_{2}\right)\right), \quad i=1,2 .
$$

In order to prove well-posedness of problem (3.6) it suffices to show that

$$
\partial \Phi(0)=\left[\begin{array}{ll}
\partial_{\rho_{1}} \Phi_{1}(0) & \partial_{\rho_{2}} \Phi_{1}(0) \\
\partial_{\rho_{1}} \Phi_{2}(0) & \partial_{\rho_{2}} \Phi_{2}(0)
\end{array}\right]
$$

generates a strongly continuous and analytic semigroup. The key role is played by the operator $\mathcal{S}$ which depends on the highest order derivatives of $\rho_{i}, i=1,2$. We have: 
Theorem 3.2. The operator $\Phi$ is analytic, i.e. $\Phi \in C^{\omega}\left(\mathcal{V}^{2},\left(h^{1+\alpha}(\mathbb{S})\right)^{2}\right)$. Given $\beta \in(0,1)$, the Fréchet derivative $\partial \Phi(0)$, seen as an unbounded operator in $\left(h^{1+\beta}(\mathbb{S})\right)^{2}$ with domain $\left(h^{4+\beta}(\mathbb{S})\right)^{2}$ generates a strongly continuous and analytic semigroup in $\mathcal{L}\left(\left(h^{1+\beta}(\mathbb{S})\right)^{2}\right)$, i.e.

$$
-\partial \Phi(0) \in \mathcal{H}\left(\left(h^{4+\beta}(\mathbb{S})\right)^{2},\left(h^{1+\beta}(\mathbb{S})\right)^{2}\right) .
$$

Proof. The regularity assumption follows directly from (3.1) and (3.2). Moreover, since the constant $\alpha$ fixed at the begining of this section was arbitrary, we may replace $\alpha$ by $\beta$ and all the assetions already established remain valid.

Let us now study the Fréchet derivative of $\Phi$ in 0 . One can easily see that the highest order terms in $\left(\rho_{1}, \rho_{2}\right)$ of $\partial \Phi(0,0)\left[\left(\rho_{1}, \rho_{2}\right)\right]$ are those obtained when differentiating the curvature operator.

Consider first $\partial_{\rho_{1}} \Phi_{1}(0)$. Since $\partial \kappa(0)[\rho]=-\rho^{\prime \prime}-\rho$ for $\rho \in \rho^{4+\alpha}(\mathbb{S})$, we may decompose

$$
\partial_{\rho_{1}} \Phi_{1}(0)\left[\rho_{1}\right]=A_{11}+B_{11},
$$

where $B_{11}$ is an operator of first order, i.e. $B_{11} \in \mathcal{L}\left(h^{2+\beta}(\mathbb{S}), h^{1+\beta}(\mathbb{S})\right)$,

$$
A_{11} \rho_{1}:=-\frac{1}{R_{1}^{2}} C_{1}(0)\left(\Delta, \operatorname{tr}_{1}, \operatorname{tr}_{2}\right)^{-1}\left(0, \rho_{1}^{\prime \prime}, 0\right), \quad \forall \rho_{1} \in h^{4+\beta}(\mathbb{S}),
$$

and $\operatorname{tr}_{i}, i=1,2$, is the trace operator with respect to $\Gamma_{i}$. We determine now a Fourier expansion for the highest order term of $\partial_{\rho_{1}} \Phi_{1}(0)\left[h_{1}\right]$. Given $\rho_{1} \in \rho^{4+\beta}(\mathbb{S})$, the function $w:=\left(\Delta, \operatorname{tr}_{1}, \operatorname{tr}_{2}\right)^{-1}\left(0, h_{1}^{\prime \prime}, 0\right)$ is the solution of the Dirichlet problem

$$
\left\{\begin{array}{rlll}
\Delta w & = & & \text { in } \Omega \\
w & =\rho_{1}^{\prime \prime} & & \text { on } \Gamma_{1} \\
w & =0 & & \text { on } \Gamma_{2} .
\end{array}\right.
$$

If we expand

$$
\rho_{1}(y)=\sum_{m} \widehat{\rho}_{1}(m) y^{m} \text { and } w(r y)=\sum_{m \in \mathbb{Z}} w_{m}(r) y^{k}
$$

for $y \in \mathbb{S}$ and $R_{2}<r<R_{1}$, we find that $w_{0}=0$, and $w_{m}$ solves, for $|m| \geq 1$, the problem

$$
\left\{\begin{array}{rl}
w_{m}^{\prime \prime}+\frac{1}{r} w_{m}^{\prime}-\frac{m^{2}}{r^{2}} w_{m} & =0 \\
w_{m}\left(R_{1}\right) & =-m^{2} \widehat{\rho}_{1}(m) \\
w_{m}\left(R_{2}\right) & =0
\end{array} \quad R_{2}<r<R_{1}\right.
$$

Hence

$$
w_{m}(r)=-\frac{R_{2}^{m} r^{-m}-R_{2}^{-m} r^{m}}{R_{2}^{m} R_{1}^{-m}-R_{2}^{-m} R_{1}^{m}} m^{2} \widehat{\rho}_{1}(m)
$$


and therewith

$$
\begin{aligned}
A_{11} \rho_{1}(y) & =-\frac{1}{R_{1}^{2}} \mathcal{C}_{1}(0) w(y)=-\frac{1}{R_{1}^{2}}\left\langle\nabla w\left(R_{1} y\right) \mid y\right\rangle=-\left.\frac{1}{R_{1}^{2}} \frac{d}{d r}(w(r y))\right|_{r=R_{1}} \\
& =-\frac{1}{R_{1}^{3}} \sum_{m \in \mathbb{Z} \backslash\{0\}} \frac{R_{1}^{|m|} R_{2}^{-|m|}+R_{1}^{-|m|} R_{2}^{|m|}}{R_{1}^{|m|} R_{2}^{-|m|}-R_{1}^{-|m|} R_{2}^{|m|}}|m|^{3} \widehat{\rho}_{1}(m) y^{m} .
\end{aligned}
$$

We proceed similarly and write $\partial_{\rho_{2}} \Phi_{1}(0)=A_{12}+B_{12}$, where $B_{12} \in$ $\mathcal{L}\left(h^{2+\beta}(\mathbb{S}), h^{1+\beta}(\mathbb{S})\right)$ and

$$
A_{12} \rho_{2}:=-\frac{1}{R_{1} R_{2}} C_{1}(0)\left(\Delta, \operatorname{tr}_{1}, \operatorname{tr}_{2}\right)^{-1}\left(0,0, \rho_{2}^{\prime \prime}\right) \quad \forall \rho_{2} \in h^{4+\beta}(\mathbb{S}) .
$$

Given $\rho_{2} \in h^{4+\beta}(\mathbb{S})$, the function $w:=\left(\Delta, \operatorname{tr}_{1}, \operatorname{tr}_{2}\right)^{-1}\left(0,0, \rho_{2}^{\prime \prime}\right)$ is the solution of linear Dirichlet problem

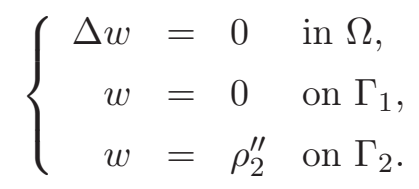

A Fourier series ansatz as we did before yields that

$$
w(r y)=-\sum_{m \in \mathbb{Z} \backslash\{0\}} \frac{R_{1}^{m} r^{-m}-R_{1}^{-m} r^{m}}{R_{1}^{m} R_{2}^{-m}-R_{1}^{-m} R_{2}^{m}} m^{2} \widehat{\rho}_{2}(m) y^{m}
$$

for all $y \in \mathbb{S}$ and $R_{2}<r<R_{1}$, provided that $\rho_{2}=\sum_{m \in \mathbb{Z}} \widehat{\rho}_{2}(m) y^{m}$. Whence,

$$
A_{12} \sum_{m \in \mathbb{Z}} \widehat{\rho}_{2}(m) y^{m}=-\frac{1}{R_{1}^{2} R_{2}} \sum_{m \in \mathbb{Z} \backslash\{0\}} \frac{2}{R_{1}^{|m|} R_{2}^{-|m|}-R_{1}^{-|m|} R_{2}^{|m|}}|m|^{3} \widehat{\rho}_{2}(m) y^{m} .
$$

We consider now the second component $\Phi_{2}$ and continue our computation following the same scheme. The second diagonal element of the matrix $\partial \Phi(0)$ may be also written as the sum $\partial_{\rho_{2}} \Phi_{2}(0)=A_{22}+B_{22}$, with $B_{22} \in \mathcal{L}\left(h^{2+\beta}(\mathbb{S}), h^{1+\beta}(\mathbb{S})\right)$ and

$$
A_{22} \rho_{2}:=-\frac{1}{R_{2}^{2}} C_{2}(0)\left(\Delta, \operatorname{tr}_{1}, \operatorname{tr}_{2}\right)^{-1}\left(0,0, \rho_{2}^{\prime \prime}\right) \quad \forall \rho_{2} \in h^{4+\beta}(\mathbb{S})
$$

Using once more the expansion for the solution of (3.8), we find out that

$$
A_{22} \sum_{m \in \mathbb{Z}} \widehat{\rho}_{2}(m) y^{m}=-\frac{1}{R_{2}^{3}} \sum_{m \in \mathbb{Z} \backslash\{0\}} \frac{R_{1}^{|m|} R_{2}^{-|m|}+R_{1}^{-|m|} R_{2}^{|m|}}{R_{1}^{|m|} R_{2}^{-|m|}-R_{1}^{-|m|} R_{2}^{|m|}}|m|^{3} \widehat{\rho}_{2}(m) y^{m} .
$$

for all $\rho_{2}=\sum_{m \in \mathbb{Z}} \widehat{\rho}_{2}(m) y^{m}$ within $h^{4+\beta}(\mathbb{S})$. Finally, $\partial_{\rho_{1}} \Phi_{2}(0)=A_{21}+B_{21}$, where $B_{21} \in \mathcal{L}\left(h^{2+\beta}(\mathbb{S}), h^{1+\beta}(\mathbb{S})\right)$ and

$$
A_{21} \rho_{1}:=\frac{1}{R_{1} R_{2}} C_{2}(0)\left(\Delta, \operatorname{tr}_{1}, \operatorname{tr}_{2}\right)^{-1}\left(0, \rho_{1}^{\prime \prime}, 0\right) \quad \forall \rho_{2} \in h^{4+\beta}(\mathbb{S}) .
$$


Since $\left(\Delta, \operatorname{tr}_{1}, \operatorname{tr}_{2}\right)^{-1}\left(0, \rho_{1}^{\prime \prime}, 0\right)$ is the solution of (3.7), we may use the expansion found at that point of the proof and obtain that

$$
A_{21} \sum_{m \in \mathbb{Z}} \widehat{\rho}_{1}(m) y^{m}=-\frac{1}{R_{1} R_{2}^{2}} \sum_{m \in \mathbb{Z} \backslash\{0\}} \frac{2}{R_{1}^{|m|} R_{2}^{-|m|}-R_{1}^{-|m|} R_{2}^{|m|}}|m|^{3} \widehat{\rho}_{1}(m) y^{m}
$$

for all functions $\rho_{1}=\sum_{m \in \mathbb{Z}} \widehat{\rho}_{1}(m) y^{m}$ in $h^{4+\beta}(\mathbb{S})$.

Let us notice that the operators $A_{i j}, 1 \leq i, j \leq 2$, found above are all Fourier multipliers, since they are of the form

$$
\sum_{m \in \mathbb{Z}} \widehat{\rho}_{1}(m) y^{m} \mapsto \sum_{m \in \mathbb{Z}} M_{k} \widehat{\rho}_{1}(m) y^{m}
$$

with symbol $\left(M_{k}\right)_{k \in \mathbb{Z}} \subset \mathbb{C}$. Using [14, Theorem 3.4], which is a theorem characterising multiplier operators between Hölder spaces by studying some generalised Marcinkiewicz conditions for the symbol of the operator, we find out that $-A_{i i} \in \mathcal{H}\left(h^{4+\beta}(\mathbb{S}), h^{1+\beta}(\mathbb{S})\right), i=1,2$, and that $A_{12}, A_{21} \in \mathcal{L}\left(h^{2+\beta}(\mathbb{S})\right)$. This may be seen form the following relations

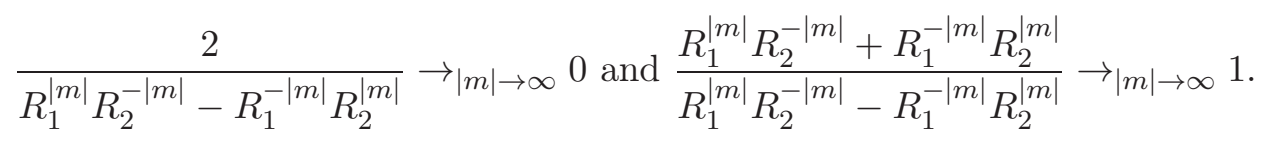

Since $h^{2+\beta}(\mathbb{S})$ is an intermidiate space,

$$
h^{2+\beta}(\mathbb{S})=\left(h^{1+\alpha}(\mathbb{S}), h^{4+\alpha}(\mathbb{S})\right)_{(1+\beta-\alpha) / 3},
$$

where $(\cdot \mid \cdot)$ denotes the interpolation functor introduced by Da Prato and Grisvard [11], we obtain form [19, Proposition 2.4.1] that the elements on the diagonal of $\partial \Phi(0)$ generate analytic semigroups, that is

$$
-\partial_{\rho_{i}} \Phi_{i} i \in \mathcal{H}\left(h^{4+\beta}(\mathbb{S}), h^{1+\alpha}(\mathbb{S})\right), i=1,2,
$$

while the elements on the secondary diagonal belong to $\mathcal{L}\left(h^{2+\beta}(\mathbb{S}), h^{1+\beta}(\mathbb{S})\right)$, that is they have lower order. We obtain then form [1, Theorem 1.6.1] that the matrix $\partial \Phi(0)$ is a generator, which completes the proof.

We give now a short proof of our second main result, Theorem 1.2.

Proof of Theorem 1.2. Let $0<\beta<\alpha$. Since $\Phi$ is analytic and $\partial \Phi(0)$ generates a strongly continuous and analytic semigroup, we find an open neighborhood $\widetilde{\mathcal{O}}$ of 0 in $h^{4+\beta}(\mathbb{S})$ such that $-\partial \Phi\left(\rho_{1}, \rho_{2}\right) \in \mathcal{H}\left(\left(h^{4+\beta}(\mathbb{S})\right)^{2},\left(h^{1+\beta}(\mathbb{S})\right)^{2}\right)$ for all $\left(\rho_{1}, \rho_{2}\right) \in \widetilde{\mathcal{O}}^{2}$. Letting $\left.\mathcal{O}:=\widetilde{\mathcal{O}} \cap h^{4+\alpha}(\mathbb{S})\right)$, we find that $-\partial \Phi\left(\rho_{1}, \rho_{2}\right) \in$ $\mathcal{H}\left(\left(h^{4+\alpha}(\mathbb{S})\right)^{2},\left(h^{1+\alpha}(\mathbb{S})\right)^{2}\right)$ is, for all $\left(\rho_{1}, \rho_{2}\right) \in \mathcal{O}^{2}$, the realisation of the operator $-\partial \Phi\left(\rho_{1}, \rho_{2}\right) \in \mathcal{H}\left(\left(h^{4+\beta}(\mathbb{S})\right)^{2},\left(h^{1+\beta}(\mathbb{S})\right)^{2}\right)$. Whence, the assumptions of [19, Theorem 8.4.1] are all satisfied and the desired assertion follows at once. 


\section{REFERENCES}

[1] H. Amann: Linear and Quasilinear Parabolic Problems, Volume I, Birkhäuser, Basel, 1995.

[2] G. B. Arfken \& H. J. Weber: "Mathematical Methods for Physicists", Elsevier Academic Press, Amsterdam, (2005).

[3] A. Borisovich \& A. Friedman: Symmetric-breaking bifurcation for free boundary problems, Indiana Univ. Math. J. 54, 927-947 (2005).

[4] H. M. Byrne \& M. A. Chaplain: Growth of nonnecrotic tumors in the presence and absence of inhibitors Math. Biosci., 130, 151-181 (1995).

[5] V. Cristini, J. Lowengrub \& Q. Nie: Nonlinear simulation of tumor growth, Journal of Mathematical Biology, 46, 191-224 (2003).

[6] S. B. CuI: Analysis of a free boundary problem modeling tumor growth, Acta Mathematica Sinica, English Series, 21 (5), 1071-1082 (2005).

[7] S. B. Cui \& J. Escher: Bifurcation analysis of an elliptic free boundary problem modelling the growth of avascular tumors, SIAM J. Math. Anal., 39 (1), 210-235 (2007).

[8] S. B. Cui \& J. Escher: Asymptotic behaviour of solutions of a multidimensional moving boundary problem modeling tumor growth, Comm. Part. Diff. Eq., 33 (4), 636-655 (2008).

[9] S. B. Cui, J. Escher \& F. Zhou: Bifurcation for a free boundary problem with surface tension modelling the growth of multi-layer tumors, J. Math. Anal. Appl., 337 (1), 443-457 (2008).

[10] S. B. Cui \& A. Friedman: Analysis of a mathematical model of the growth of necrotic tumors, J. Math. Anal. Appl., 255, 636-677 (2001).

[11] G. Da Prato \& P. Grisvard: Equations d'évolution abstraites nonlinéaires de type parabolique, Ann. Mat. Pura Appl., 120 (1979), 329-326.

[12] J. Escher \& A-V. Matioc: Radially symmetric growth of nonnecrotic tumors, to appear in Nonlinear Differential Equations and Applications.

[13] J. Escher \& A-V. Matioc: Well-posedness and stability analysis for a moving boundary problem modelling the growth of nonnecrotic tumors, submitted.

[14] J. Escher \& B.--V. MATioc: A moving boundary problem for periodic Stokesian Hele-Shaw flows, Interfaces Free Bound., 11 (2009), 119-137.

[15] A. Friedman \& F. Reitich: Analysis of a mathematical model for the growth of tumors, J. Math. Biol., 38, 262-284 (1999).

[16] D. Gilbarg \& T. S. Trudinger: Elliptic Partial Differential Equations of Second Order, Springer-Verlag, New York, 1998.

[17] H. P. Greenspan: On the growth and stability of cell cultures and solid tumors, J. Theor. Biol., 56, 229-242 (1976).

[18] H. P. Greenspan: Models for the growth of a solid tumor by diffusion, Stud. Appl. Math. , LI(4), 317-340 (1972).

[19] A. Lunardi: Analytic Semigroups and Optimal Regularity in Parabolic Problems, Birkhäuser, Basel, 1995. 
Institut für Angewandte Mathematik, Leibniz Universität Hannover, Welfengarten 1, 30167 Hannover, Germany.

E-mail address: escher@ifam.uni-hannover.de

Institut für Angewandte Mathematik, Leibniz Universität Hannover, WelfenGarten 1, 30167 Hannover, Germany.

E-mail address: matioca@ifam.uni-hannover.de

Institut für Angewandte Mathematik, Leibniz Universität Hannover, Welfengarten 1, 30167 Hannover, Germany.

E-mail address: matioca@ifam.uni-hannover.de 